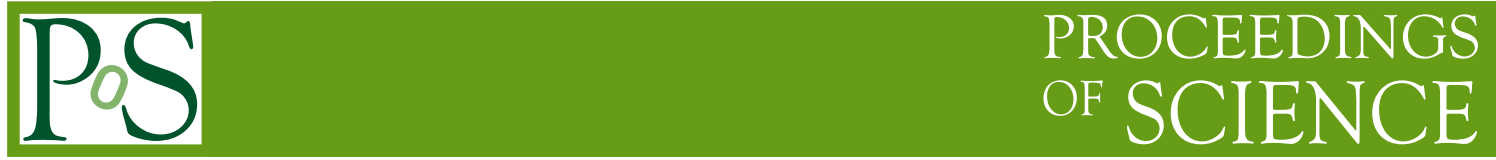

\title{
Theory of indirect dark matter detection
}

\author{
Stefano Profumo* \\ Santa Cruz Institute for Particle Physics and Department of Physics, \\ University of California, Santa Cruz CA 95064 \\ E-mail: profumo@scipp.ucsc.edu
}

Recent cosmic ray data, notably from the Pamela and Fermi satellites, indicate that previously unaccounted-for powerful sources in the Galaxy inject high-energy electrons and positrons. Interestingly, this new source class might be related to new fundamental particle physics, and specifically to pair-annihilation or decay of galactic dark matter. I will discuss how this exciting scenario is constrained by Fermi gamma-ray observations, and which astrophysical source counterparts could also be responsible for the high-energy electron-positron excess. In particular, I will review the case for nearby mature pulsars, and the search for anisotropies in the electron-positron arrival directions as a diagnostic between the pulsars and the dark matter scenarios.

25th Texas Symposium on Relativistic Astrophysics

December 6-10, 2010

Heidelberg, Germany

\footnotetext{
* Speaker.
} 


\section{Introduction}

The indirect detection of particle dark matter hinges on the possibility of detecting the debris of pair-annihilations of dark matter particles in the halo of the Milky Way or of external galaxies. In most particle dark matter models, this possibility exists as a result of the feeble, but existent, probability that dark matter pair-annihilates into Standard Model particles, and that this mechanism is responsible for the present dark matter density as a result of freeze-out from the thermal bath filling the early Universe.

Indirect detection is based on one's ability to disentangle ordinary astrophysical background emissions from peculiar signatures that might be associated with the annihilation event described above. Possible diagnostics include antimatter (positrons, antiprotons and antideuterons), gamma rays, high-energy neutrinos and the secondary emission from high-energy electrons and positrons.

At present, the most interesting aspects of indirect detection under discussion and investigation include the "WMAP haze", first discussed in [1], the EGRET GeV excess (that recently was shown to not be confirmed by Fermi data), the recent limits on the gamma-ray emission from local clusters of galaxies and from nearby dwarf galaxies satellites of the Milky Way reported by Fermi, and the series of puzzling results involving the local flux of electrons and positrons $\left(e^{+} e^{-}\right)$. In my contribution, I will focus on the latter.

The Pamela experiment has recently reported [2] an excess of high-energy (10-100 GeV) positrons over the standard expectation from diffuse galactic cosmic ray secondary models (i.e. models where positrons result from inelastic collisions of primary protons on the intra-galactic gas nuclei). This is a generic prediction for dark matter pair-annihilation, since if dark matter is self-C-conjugate, its annihilation produces as many positrons as electrons, therefore enhancing the positron fraction, with particles of energies close to the dark matter particle mass. In turn, the latter is in the ten's to hundred's of GeV range in the context of the best motivated particle models.

Additionally, a balloon-borne experiment, ATIC, also reported an anomalous "bump" in the total flux of $e^{+} e^{-}$, at energies of a few hundreds GeV [3]. Again, this is in principle consistent with galactic dark matter annihilation, and it would imply a large mass and a very large pair annihilation cross section for dark matter.

At odds with the ATIC result, the Fermi-LAT (Large Area Telescope) Collaboration reported (with much larger statistics) a featureless $e^{+} e^{-}$spectrum up to TeV energies [4]. Fermi data are entirely consistent with a standard diffuse galactic cosmic-ray model, where electrons are accelerated by continuously distributed astrophysical sources. Fits to the Fermi data imply harder (i.e. smaller) injection spectral indexes: depending on the diffusion model, best fit injection spectral indexes range between 2.3 and 2.4, as opposed to previous models with 2.54. The ATIC anomalous bump is therefore excluded by Fermi-LAT, which collected in a few months of operations more than 2 orders of magnitude more high-energy $e^{+} e^{-}$events than all ATIC flights combined.

A residual feature in the several hundred $\mathrm{GeV}$ range has been claimed in association with the Fermi-LAT data. There is convincing evidence that this is not the case, once account is taken of (1) the possible existence of cut-offs in the cosmic ray source spectra, and of (2) the fact that the diffusion radius at those energies is comparable to the mean separation between sources, and therefore stochasticity effects (not included in ordinary diffuse cosmic ray models) can account for the presence of a bump at the energy where the Fermi-LAT $e^{+} e^{-}$spectrum appears to over-shoot 
the prediction of ordinary cosmic ray models. The same two effects mentioned above can account for the softer spectral index implied by the results reported by the H.E.S.S. atmospheric Cherenkov Telescope [5] at $e^{+} e^{-}$energies above a TeV.

The relatively hard (compared to pre-Fermi measurements and models) $e^{+} e^{-}$spectrum measured by the LAT implies however conclusively that the Pamela positron fraction data cannot have a purely secondary origin [6]: an additional primary positron source is required to match the highenergy positron fraction data reported in [2]. The new Fermi data make the Pamela result on the positron fraction in even more striking contrast with the standard diffuse galactic cosmic ray expectation.

\section{The source of high energy cosmic-ray electrons and positrons}

Two possible primary $e^{+} e^{-}$source classes have been widely discussed in the literature: pulsars, producing $e^{+} e^{-}$pairs in their magnetosphere, and galactic dark matter annihilation or decay. In addition, the possibility that secondary $e^{+} e^{-}$are accelerated in situ, i.e. at the location where primary cosmic rays are produced, has also been entertained [7]. While this latter scenario would imply striking features in cosmic ray secondary-to-primary ratios (for instance a steeply increasing antiproton-to-proton ratio above $100 \mathrm{GeV}$ [8]; an upturn in that energy range is also expected for the $\mathrm{Ti} / \mathrm{Fe}$ and for the $\mathrm{B} / \mathrm{C}$ ratios [8]), it is less clear if conclusive diagnostics exist that would allow one to discriminate between the pulsar and the dark matter origin of high energy galactic $e^{+} e^{-}$.

Here, we thus intend to address the following question: what is the impact of the Fermi-LAT data on understanding the origin of the extra $e^{+} e^{-}$? In [6] we showed examples of excellent fits to both Fermi and Pamela data with known (ATNF catalogue) nearby, mature pulsars and with a single, nominal choice for the $e^{+} e^{-}$injection parameters. Scanning on poorly known pulsar parameters, Ref. [6] concluded that under reasonable assumptions, electron/positron emission from pulsars offers a viable interpretation of Fermi CRE data which is also consistent with the H.E.S.S. and Pamela results.

A possible issue with the pulsar interpretation arises from the needed degree of efficiency in converting the pulsars' spin-down luminosity into the energy associated with cosmic-ray electrons and positrons after being injected into the inter-stellar medium. Observations of pulsar wind nebulae with the Fermi Telescope, for instance, seem to point to efficiencies smaller than one percent, as opposed to the tens of percent needed to explain the Pamela results with known radio pulsars.

We recently pointed out, however, in Ref. [15], how the incompleteness of pulsar radio catalogues due to the simple geometric consideration of the so-called lighthouse effect might affect our knowledge of the main contributors to the local $e^{+} e^{-}$flux. In this respect, Ref. [15] discovered that radio-quiet gamma-ray pulsars detected by the Fermi Telescope, including several discovered with blind searches, significantly contribute to the local $e^{+} e^{-}$flux, possibly alleviating the mentioned tension.

The dark matter interpretation attracted a very large scientific interest. Numerous scenarios, which we won't review here, have been envisioned to overcome the obvious issues related to a dark matter origin of the high-energy $e^{+} e^{-}$data (e.g. the absence of an antiproton excess and the large required pair-annihilation cross section): as the late Cambridge astronomer Roderick Redman put 

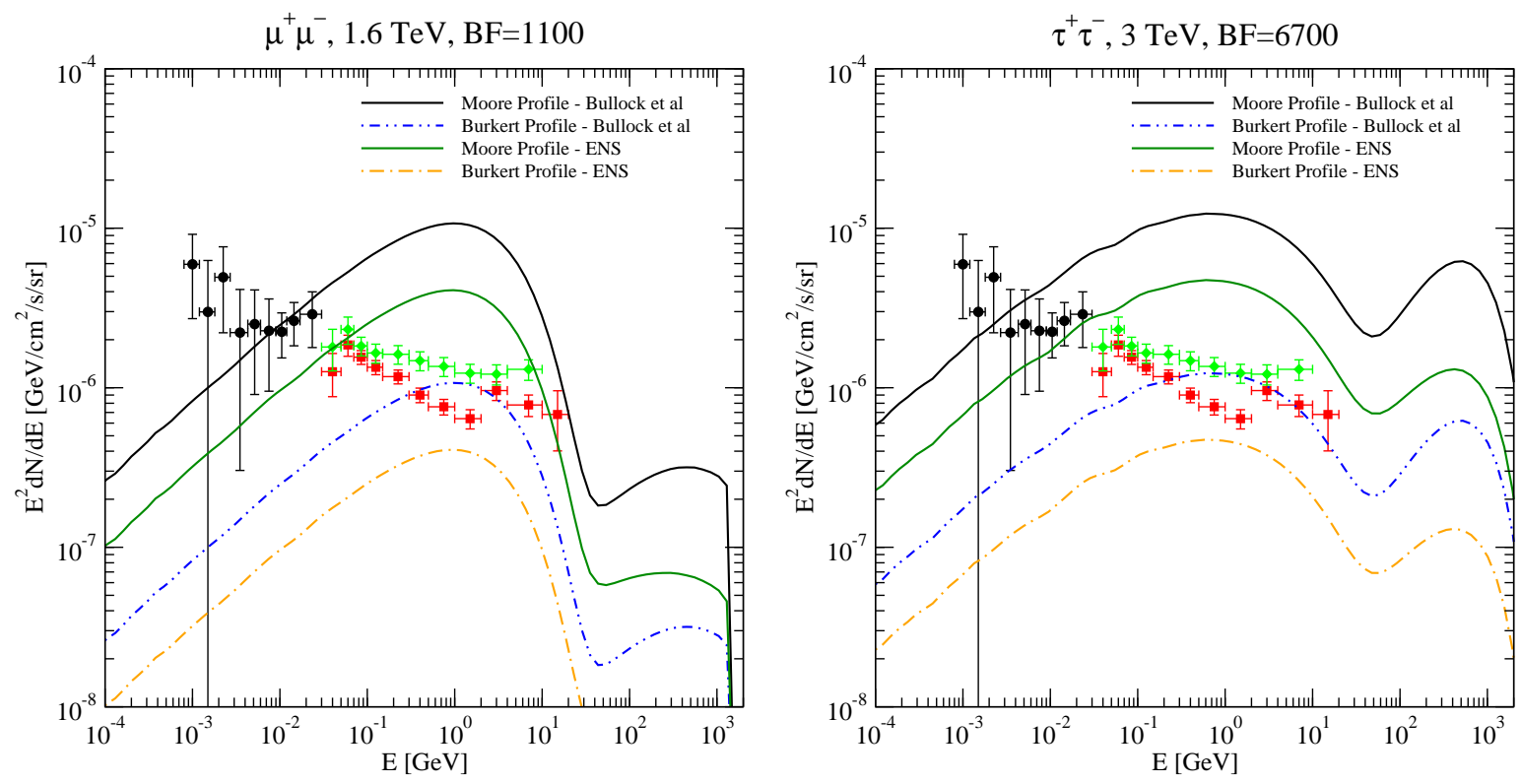

Figure 1: The dependence of the dark matter all-redshift all-halo annihilation emission on the structure formation and halo model setup, for particle dark matter models that offer an explanation to the Pamela positron excess and that are compatible with the Fermi-LAT electron-positron data. The red and green data points refer to two determinations of the extragalactic isotropic gamma-ray flux from the EGRET data (see Ref. [9] and [10]), while the black data points refer to COMPTEL data [11]. See Ref. [12] for details.

it, "any competent theoretician can fit any given theory to any given set of facts". Fermi-LAT data are impacting and will constrain a dark matter interpretation at least in the following ways:

1. There is a much weaker rationale to postulate a dark matter mass in the $0.3-1 \mathrm{TeV}$ range (a so-called "ATIC bum") motivated by the $e^{+} e^{-}$spectrum;

2. If the Pamela positron excess is from dark matter annihilation or decay, Fermi $e^{+} e^{-}$data set stringent constraints on such interpretation;

3. Even neglecting the poistron fraction data reported by Pamela, Fermi $e^{+} e^{-}$data are useful to put limits on rates for particle dark matter annihilation or decay;

4. It is possible, however, to find dark matter scenarios that provide a reasonable fir to the Pamela positron fraction data and that are consistent with the new Fermi-LAT $e^{+} e^{-}$data [6].

What is the role of Fermi to assess the origin of high-energy $e^{+} e^{-}$? Probably, further accurate spectral information on the $e^{+} e^{-}$flux as a function of energy will not conclusively help disentangle the pulsar versus dark matter origin of the additional primary $e^{+} e^{-}$source needed to explain the Pamela positron fraction data. The existence of a local $e^{+} e^{-}$source can however be tested with Fermi-LAT data, by comparing the Inverse Compton and Bremsstrahlung emission predicted from the measured $e^{+} e^{-}$spectrum with actual diffuse gamma-ray data. Detailed knowledge on local gamma-ray pulsars will enormously further our grasp of their electro-magnetic emission mechanisms and properties, and gauge the $e^{+} e^{-}$emission as well. 

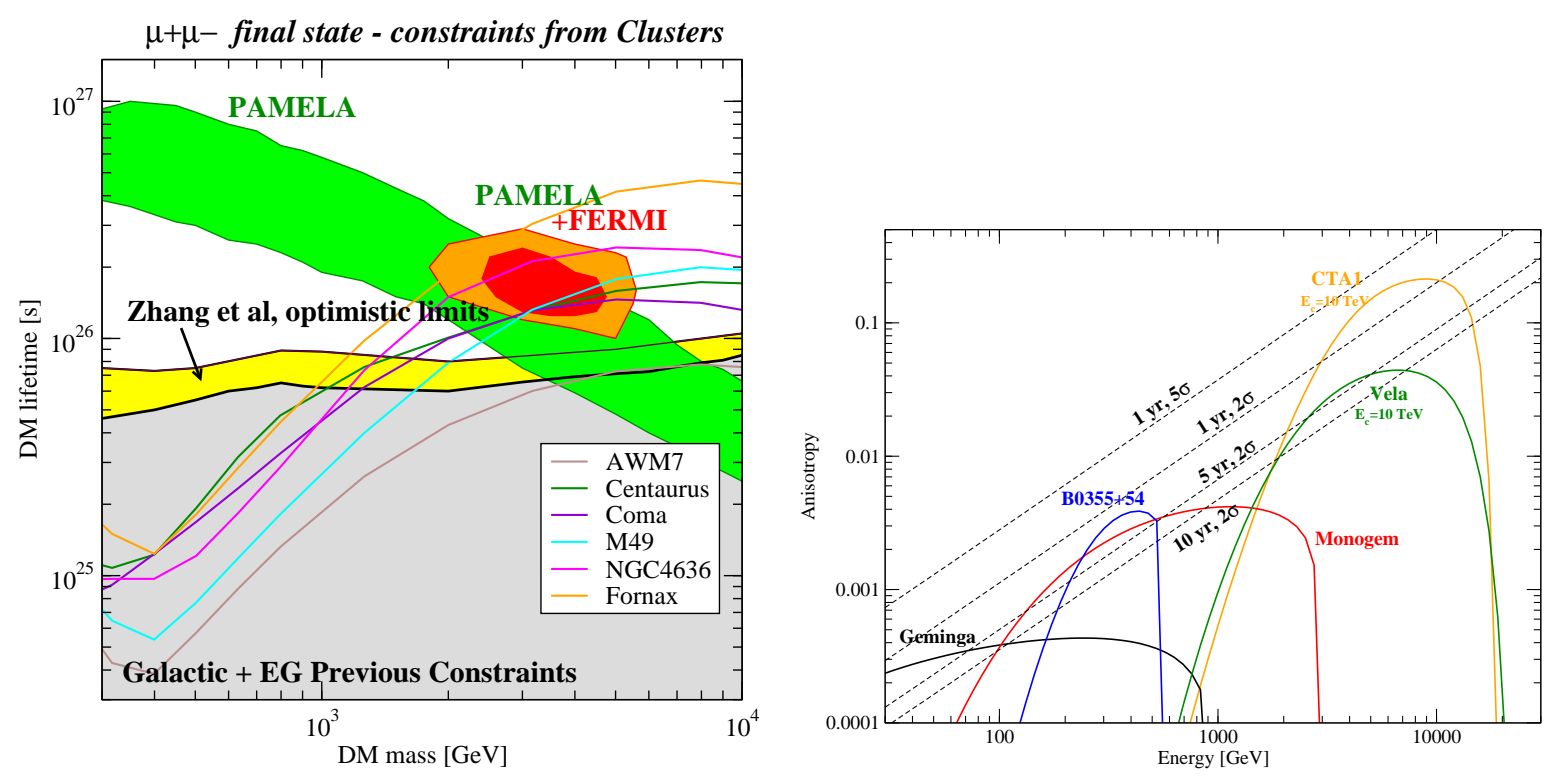

Figure 2: Left panel: Constraints on the decay lifetime as a function of mass from clusters of galaxies for a $\mu^{+} \mu^{-}$final state based on the $95 \%$ confidence level upper limits on the gamma-ray flux from 11 months of Fermi-LAT observations. The grey shaded region shows an example of previous constraints which consider the expected diffuse dark matter decay signal from the Galactic halo and unresolved extragalactic dark matter; the yellow shaded region in the right panel shows more optimistic limits obtained after subtraction of a model for the Galactic diffuse emission. We also show the regions of parameter space fitting the observed cosmic-ray anomalies. Right panel: Predictions for the anisotropy in the arrival direction of cosmic ray leptons from nearby pulsars, defined as $\left(I_{f}-I_{b}\right) /\left(I_{f}+I_{b}\right)$, where $I_{f}$ is the total number of events from the emisphere in the direction of the pulsar, and $I_{b}$ that from the opposite emisphere. See Ref. [14] for details

As far as dark matter is concerned, cross-constraints on models with large pair-annhilation cross sections can be cast with orthogonal probes to $e^{+} e^{-}$, such as e.g. gamma-ray emission. Ref. [12] for instance showed that models favored by a dark matter annihilation of the Pamela positron fraction data over-produce gamma rays via inverse-Compton emission at all redshifts and from annihilation in all halos. We consider in fig. 1 two examples of particle dark matter setups that would fit the Pamela positron fraction data and be compatible with the measured total $e^{+} e^{-}$flux. The four lines correspond to various assumptions on structure formation (including the concentration of halos as a function of mass and redshift, and the matter power spectrum) and on the density profile of halos (assumed to be universal). As evident, even with data from EGRET (red and green data points) and COMPTEL (black points) the extragalactic flux of gamma rays from inverse Compton scattering of CMB radiation is in conflict with observations. Similar conclusions apply to the possibility that most of the detected $e^{+} e^{-}$originate from a local nearby bright dark matter clump, which would be easily detectable in gamma rays with the LAT, as showed e.g. in [13].

We also recently showed in Ref. [16] that the dark matter decay scenario is severly constrained by Fermi-LAT non-observations of nearby massive galaxy clusters. Albeit theoretically motivated by, for instance, dimension-6 GUT-scale operators, a dark matter particle decaying with a lifetime on the order of $10^{26} \mathrm{~s}$ into, for isntance, muon pairs would produce enough energetic 
$e^{+} e^{-}$(effectively confined within the cluster volume) to induce copious gamma ray emission from inverse Compton up-scattering of cosmic microwave background photons. Fig. 2, left, illustrates the constrained we obtained in [16].

Finally, the Fermi-LAT collaboration is currently pursuing a dipole anisotropy search in the arrival direction of high-energy $e^{+} e^{-}$, that might potentially indicate a preferred local source direction and identify it with an existing astrophysical object, such as a young mature pulsar. We show in fig, 2, right, the predicted anisotropy (defined as usual as $\left(I_{f}-I_{b}\right) /\left(I_{f}+I_{b}\right)$, where $I_{f}$ is the total number of events from the emisphere centered on the direction of the pulsar, and $I_{b}$ that of events from the opposite emisphere), as a function of energy, produced by the pulsars we considered in Ref. [14]. The Fermi Collaboration recently reported a preliminary null result on the anisotropy of the $e^{+} e^{-}$arrival directions [17]. Unfortunately, the level of anisotropy currently constrained by this result does not achieves the one predicted by a nearby pulsar that would explain the Pamela excess [17]. We also note that even the detection of an anisotropy in the $e^{+} e^{-}$data might not be conclusive in favor of pulsars as the origin of the positron excess: a nearby, dense and massive dark matter clump might also produce a comparable level of anisotropy.

\section{Conclusions}

In summary, Fermi $e^{+} e^{-}$data indicate a hard high-energy spectrum, which is perfectly compatible with Diffuse Galactic Cosmic Ray origin, but which, including Pamela data, rules out a purely secondary diffuse cosmic-ray origin for the positron excess. Pulsars appear to be strong candidates as primary local positron sources, and while dark matter annihilation (or decay) is not entirely ruled out by Fermi data as a possible high-energy $e^{+} e^{-}$source, this possibility is currently under close scrutiny with other indirect dark matter search channels, in particular gamma rays.

\section{Acknowledgements}

This work and SP's participation to the Texas 2010 conference have been partly supported by US Department of Energy Contract DE-FG02-04ER41268 and by an Outstanding Junior Investigator Award, by NASA Grant Number NNX08AV72G and by NSF Grant PHY-0757911.

\section{References}

[1] D. P. Finkbeiner, arXiv:astro-ph/0409027.

[2] O. Adriani et al. [PAMELA Collaboration], Nature 458 (2009) 607 [arXiv:0810.4995 [astro-ph]].

[3] J. Chang et al., Nature 456 (2008) 362.

[4] A. A. Abdo et al. [The Fermi LAT Collaboration], Phys. Rev. Lett. 102 (2009) 181101 [arXiv:0905.0025 [astro-ph.HE]].

[5] F. Aharonian et al. [The H.E.S.S. Collaboration] arXiv:0905.0105 [astro-ph.HE].

[6] D. Grasso et al. [FERMI-LAT Collaboration], Astrop. Phys. 32 (2009) Issue 2, 140, [arXiv:0905.0636 [astro-ph.HE]].

[7] P. Blasi, Phys. Rev. Lett. 103 (2009) 051104 [arXiv:0903.2794 [astro-ph.HE]]. 
[8] P. Blasi and P. D. Serpico, Phys. Rev. Lett. 103 (2009) 081103 [arXiv:0904.0871 [astro-ph.HE]].

[9] P. Sreekumar et al. [EGRET Collaboration], Astrophys. J. 494 (1998) 523 [arXiv:astro-ph/9709257].

[10] A. W. Strong, I. V. Moskalenko and O. Reimer, Astrophys. J. 613 (2004) 956 [arXiv:astro-ph/0405441].

[11] G. Weidenspointner et al., American Institute of Physics Conference Series, 510 (2000) 467.

[12] S. Profumo and T. E. Jeltema, JCAP 0907 (2009) 020 [arXiv:0906.0001 [astro-ph.CO]].

[13] P. Brun, T. Delahaye, J. Diemand, S. Profumo and P. Salati, Phys. Rev. D 80 (2009) 035023 [arXiv:0904.0812 [astro-ph.HE]].

[14] S. Profumo, arXiv:0812.4457 [astro-ph].

[15] L. Gendelev, S. Profumo, M. Dormody, JCAP 1002 (2010) 016 [arXiv:1001.4540 [astro-ph.HE]].

[16] L. Dugger, T. E. Jeltema, S. Profumo, [arXiv:1009.5988 [astro-ph.HE]].

[17] M. Ackermann et al. [ Fermi LAT Collaboration ], Phys. Rev. D82 (2010) 092003 [arXiv:1008.5119 [astro-ph.HE]]. 